\title{
Das Risiko ist erhöht, aber warum?
}

\author{
Krebserkrankungen treten bei Diabetikern gehäuft auf. Deshalb sollten sie die entspre- \\ chenden Angebote zur Früherkennung unbedingt wahrnehmen. Pathogenetisch dürften \\ sowohl die Insulinresistenz mit konsekutivem Hyperinsulinismus als auch die Hyperglyk- \\ ämie beteiligt sein, woraus sich der Stellenwert einer optimalen Stoffwechseleinstel- \\ lung auch im Hinblick auf die Verhinderung maligner Erkrankungen ergibt.
}

Diabetes mellitus erhöht die Inzidenz vieler Krebserkrankungen, das gilt für das Non-Hodgkin-Lymphom, das Mamma-, das kolorektale, das Harnblasen-, das Endometrium- und besonders für das Pankreas- und hepatozelluläre Karzinom $[1,2]$ als gesichert. „Nur beim Prostatakarzinom wirkt der Diabetes protektiv, da übergewichtige Patienten mit metabolischem Syndrom oft einen Hypogonadismus haben und der niedrige Testosteronspiegel der Manifestation dieses Karzinoms entgegenwirkt", sagte Prof. Norbert Stefan von der Uniklinik Tübingen.

Eine der häufigsten Ursachen des hepatozellulären Karzinoms ist die Nicht-alkoholische Fettleberhepatitis (NASH). Die Inzidenz dieser Erkrankung hat in den letzten 10 bis 15 Jahren deutlich zugenommen. Die chronischen Hepatitiden als Ursache des Leberkarzinoms sind dagegen etwa gleich häufig geblieben [3]. „Die Auslöser für NASH und somit das Leberkarzinom bei Patienten mit Adipositas und Diabetes sind die Überernährung, der Bewegungsmangel, das ungünstige Profil von Fettsäuren, Adipokinen und Zytokinen und auch eine genetische Prädisposition", so Stefan. Diese Faktoren führten zunächst zu einer benignen Fettleber, die im Verlauf in eine NASH übergehe, was wiederum zur Zirrhose und dem Karzinom führen könne [4]. Sowohl die Insulinresistenz mit konsekutivem Hyperinsulinismus als auch die Hyperglykämie selbst seien bei der malignen Entartung beteiligt, wobei aber das erhöhte Krebsrisiko wie auch die makro- und mikrovaskulären Komplikationen schon bereits vor der Manifestation des Diabetes bestehen. „Gerade die Nüchternglukose korreliert sowohl mit dem Risiko eines vaskulären als auch des malignen Todes", so Stefan.

Bei Diabetikern, und zwar beim Typ-1 wie beim Typ-2, besteht auch ein deutlich erhöhtes Risiko für die Entwicklung eines Pankreaskarzinoms, wobei der Verlauf bei Diabetikern meist sehr ungünstig ist [5]. Deshalb sollte man bei ihnen nach dieser Erkrankung fahnden. „Häufig sind es Patienten mit anhaltend erhöhten Lipasewerten, sodass sich die Lipase zum Screening eignet", so Stefan. Andererseits erhöhe ein Pankreaskarzinom auch die Diabetesinzidenz. Eine operative Entfernung der Tumoren gehe dann oft mit einer Verbesserung der Blutzuckerstoffwechsellage einher. Dies wird erklärt durch die Beobachtung, dass Pankreaskarzinome Hormone bilden, die die Insulinsekretion hemmen und die Insulinresistenz verstärken [6].

\section{Gibt es Therapieansätze?}

Doch wie kann man sich die kanzerogene Wirkung des Insulins bzw. der IGFs erklären? Diese Hormone wirken einmal über Rezeptoren für die metabolische Wirkung. Zudem werden aber auch Rezeptoren aktiviert, die bei der Proliferation, also bei einem potenziell zur malignen Entartung führenden Signalling, eine Rolle spielen. „Daraus ergeben sich auch Therapieansätze“, so Stefan. Mit Metformin, aber auch Pioglitazon lassen sich nämlich solche Signalkaskaden hemmen. In Metaanalysen konnte für diese beiden Substanzen eine günstige Wirkung im Hinblick auf das Malignitätsrisiko bei Diabetikern nachgewiesen werden. So wird mit Metformin das Risiko für ein hepatozelluläres Karzinom [7] und mit Pioglitazon für ein kolorektales Karzinom günstig beeinflusst. Auch konnte in einer Beobachtungsstudie gezeigt werden, dass eine zusätzliche Gabe von Metformin bei Patienten mit einem chemotherapeutisch behandelten Pankreaskarzinom die Prognose verbessert [8]. „Deshalb sollte man bei Diabetikern immer soweit möglich Metformin einsetzen“, so Stefan. Auch ein regelmäßiges Bewegungstraining senkt die Mortalitätsrate nicht nur im Hinblick auf kardiovaskuläre Ereignisse, sondern auch bzgl. maligner Erkrankungen [9].

\section{Koloskopie vor Beginn der Insulintherapie}

Angesichts des erhöhten Krebsrisikos sollten Diabetiker alle Möglichkeiten der Vorsorge wahrnehmen. Dies gilt insbesondere für die Vorsorgekoloskopie, zumal das Ausmaß des Übergewichts mit dem Risiko für ein kolorektales Karzinom korreliert. Außerdem ist der Verlauf dieser Erkrankung bei Adipösen ungünstiger. „Eine Koloskopie sollte bei Typ-2-Diabetikern unabhängig vom Alter immer vor Einleitung einer Insulintherapie durchgeführt werden", so Prof. Jürgen Riemann, Ludwigshafen. Neuere Erkenntnisse sprechen dafür, dass auch Patienten mit einem bariatrischen Eingriff ein erhöhtes Risiko tragen, wobei der ursächliche Mechanismus bisher noch nicht bekannt ist.

Dr. med. Peter Stiefelhagen

\footnotetext{
Literatur:

. Vignen et al. Endo Rel Cancer 2009

2. Stefan et al. DMW 2012

. Dyson et al. J of Hepat 2014

4. Stefan et al. The Lancet D \& E 2013

5. Stevens et al. J Cancer 2007

6. Permert et al. N Engl J Med 1994

7. Zhang et al. JCEM 2012

8. Choi et al. Cancer Research Treat 2015

9. Hakem et al. NEJM 1995
}

Quelle: Diabetologie grenzenlos, 20.2.2016, Unterschleißheim bei München 